\title{
Dynamic User-Oriented QoS Specification and Mapping for Multimedia Differentiation Services
}

\author{
Hsu-Yang Kung and Fu-Wen Kuo \\ Department of Management Information Systems, National Pingtung University of Science and Technology, 91207 \\ Taiwan
}

\begin{abstract}
Summary
This paper proposes the Dynamic User-Oriented QoS Framework (DUQF), which provides the effective adaptation control schemes for dynamic QoS specification and mapping based on the combination of DiffServ and IntServ network architecture. A DUQF network consists of a DiffServ WAN and lots of attached IntServ LANs that connect to each other via a RSVP-aware router and a differentiation edge router, respectively. In an IntServ LAN, we designed the User-oriented Dynamic QoS Specification and Mapping (UDQSM) system to adaptively achieve QoS specification and mapping at the application layer. In the DiffServ of DUQF, we proposed the Dynamic QoS Queue Mapping (DQ2M) algorithm to increase the utilization of network resources and find the most appropriate service class that the media packets should map in an edge router of the DiffServ network.
\end{abstract}

Key words:

Multimedia Communications, QoS Specification and Mapping, Queuing Management, Multimedia Differentiation

\section{Introduction}

With the more emergence of adaptation application and various QoS capabilities caused by the heterogeneous networks, the need to satisfy the multimedia applications with the QoS provision is urgently required. Usually, the networks providing the Differentiated Service (DiffServ) and/or Integrated Service (IntServ) can achieve QoS applications [11]. Using the RSVP mechanism of the IntServ, the network can make the resource reservation for each multimedia flow and achieves the QoS requirement [8]. However, the scalability of the IntServ network is severely restricted especially deployed in the WAN environment. In order to achieve scalability, the DiffServ network differentiates media packets into limited service classes (queues) [5]. According to the definition of the DiffServ architecture, the network resources are graded into expedited forwarding (EF) and assured forwarding (AF) to provide diverse requirements of applications in order to increase the scalability of Integrated Service
(IntServ). However, there are two critical problems arose in the DiffServ/IntServ QoS networks [2, 6, 7]. (i) The traditional way to differentiate services is based on the viewpoint of the network provider. It always results in the gap between application QoS and network QoS. (ii) The static resources allocation of the priority queues of DiffServ networks has the impacts on insufficient resources utilization when there is a burst in a certain priority queue while there is starvation in other queues.

There are many QoS parameters such as bandwidth, delay, jitter and lost rates etc. It is necessary to simplify and classify the QoS parameters [1, 4]. Various applications differ from sensitivity to various QoS parameters. For example, the audio medium is sensitive to delay and jitter but the text medium always requires good reliability. It is also important to classify QoS types based on the media and applications in use [5]. After the QoS specification, a dynamic mapping mechanism is needed to map the abstract application QoS to the network level parameters and the reserved resources dynamically satisfy the user QoS variations during the media presentation [9, 10]. Therefore, the essential considerations in achieving a multimedia QoS application are: (i) the user-oriented QoS specification, which provides a friendly QoS specification tool for users at the application layer, and the QoS mapping dynamically translating QoS parameters between the application layer and network layer of users, and (ii) the dynamic queue mapping, which can adaptively maximize the utilization of the queues and network resources based on a DiffServ network.

In this paper, we proposed the Dynamic UserOriented QoS Framework (DUQF), which is consists of a DiffServ WAN and lots of attached IntServ LANs to achieve the effective adaptation control schemes for dynamic QoS specification and mapping. The Useroriented Dynamic QoS Specification and Mapping (UDQSM) system is proposed to adaptively achieve QoS specification and mapping at the application layer in an IntServ LAN. Furthermore, to increase the utilization of 
network resources and find the most appropriate service class that the media packets should map, the Dynamic QoS Queue Mapping ( $\left.\mathrm{DQ}^{2} \mathrm{M}\right)$ algorithm is proposed.

The organization of the rest of this paper is as follows. In Section 2, we will introduce the principles of designing a user-friendly interface for QoS specification. In Section 3 , the UDQF architecture and dynamic specification and mapping schemes are described. Section 4 describes the proposed $D Q^{2} M$ algorithm. Finally we will present our conclusion in Section5.

\section{User Interface Design for QoS Specification}

The objective of the designed UI is to satisfy the user perceived requirements with conveniently choosing appropriated QoS parameters and service types based on the media type and application. Table 1 lists four application classifications, which present in the designed QoS UI. For advanced QoS specification, the QoS parameters are also divided into media control parameters and traffic control parameters in Table 2. Especially, the media control parameters focus on the most popular MPEG format.

To satisfy various kinds of users, the UI provides three QoS specification modes, which are the Easy mode, the Qualitative mode, and the Quantitative mode. Inexperienced users are suggested to use the Easy mode because it only provides users four service types depending on the applications. The Qualitative and Quantitative modes provide users with various QoS parameters for quality and quantity specifications, respectively. For example, the qualitative QoS for audio specification gives three grades, which are CD quality, FM quality and normal quality. The quantitative QoS mode will let the user to input numeric values for the precise QoS specification.

Table 1: Application classifications
\begin{tabular}{|c|c|c|}
\hline $\begin{array}{c}\text { Service } \\
\text { Types }\end{array}$ & Classifications & Description \\
\hline Premium & $\begin{array}{c}\text { VoD } \\
\text { (video and audio) }\end{array}$ & $\begin{array}{c}\text { High bandwidth } \\
\text { and low delay }\end{array}$ \\
\hline Gold & $\begin{array}{c}\text { Telephony } \\
\text { (audio only) }\end{array}$ & $\begin{array}{c}\text { Better bandwidth } \\
\text { and low delay }\end{array}$ \\
\hline Silver & WWW, Telnet & $\begin{array}{c}\text { Low bandwidth } \\
\text { and better delay }\end{array}$ \\
\hline Bronze & Mail, FTP & $\begin{array}{c}\text { Low bandwidth } \\
\text { and normal delay }\end{array}$ \\
\hline
\end{tabular}

Table 2: QoS parameters classifications

\begin{tabular}{|c|l|}
\hline $\begin{array}{c}\text { Parameter } \\
\text { classification }\end{array}$ & \multicolumn{1}{|c|}{ Description } \\
\hline $\begin{array}{c}\text { Media control } \\
\text { parameters }\end{array}$ & $\begin{array}{l}\text { Sampling rate, sampling bit, } \\
\text { DCT layering, frame rate, } \\
\text { resolution, quantization, GOP } \\
\text { format }\end{array}$ \\
\hline $\begin{array}{c}\text { Traffic control } \\
\text { parameters }\end{array}$ & $\begin{array}{l}\text { bandwidth, peak rate, delay, lost } \\
\text { rate, bit error rate, jitter, security, } \\
\text { failure notification, protocols }\end{array}$ \\
\hline
\end{tabular}

\section{User-Oriented Dynamic QoS Framework}

As depicting in Figure 1, the traditional QoS specification and mapping are static and straightforward. The static QoS specification strategy may cause two problems. (1) Inexperienced users can't appropriately specify QoS requirements at the application layer. The static QoS specification only allows users specify quantitative values of the network QoS parameters, while most inexperienced users don't know the meaning of the listed QoS parameters. It may result in a specifying gap between the application layer and network layer. (2) The inaccuracy is derived from QoS mapping between the user layer and network layer.

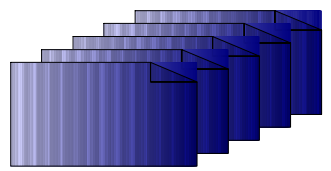

User Specify QoS Parameters

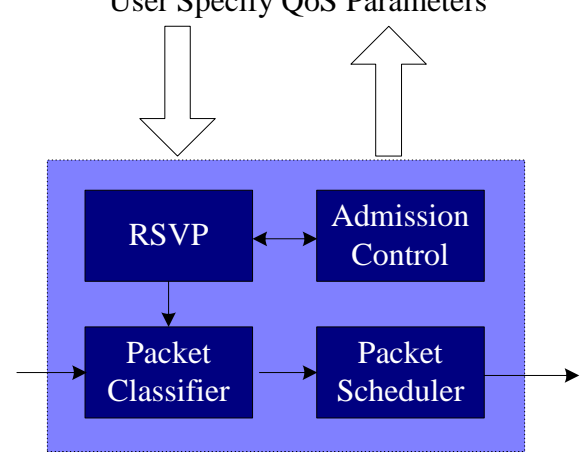

Fig. 1 Static QoS specification strategy.

To achieve the effective adaptation control for dynamic QoS specification and mapping, the Dynamic User-Oriented QoS Framework (DUQF) is proposed. As depicting in Figure 2, a DUQF consists of a DiffServ WAN and lots of attached IntServ LANs that connect to each other via a RSVP-aware router and a differentiation edge router, respectively. In an IntServ LAN, we designed the User-oriented Dynamic QoS Specification and Mapping (UDQSM) system to adaptively achieve QoS 
specification and mapping at the application layer. The

UDQSM system is composed of three components.

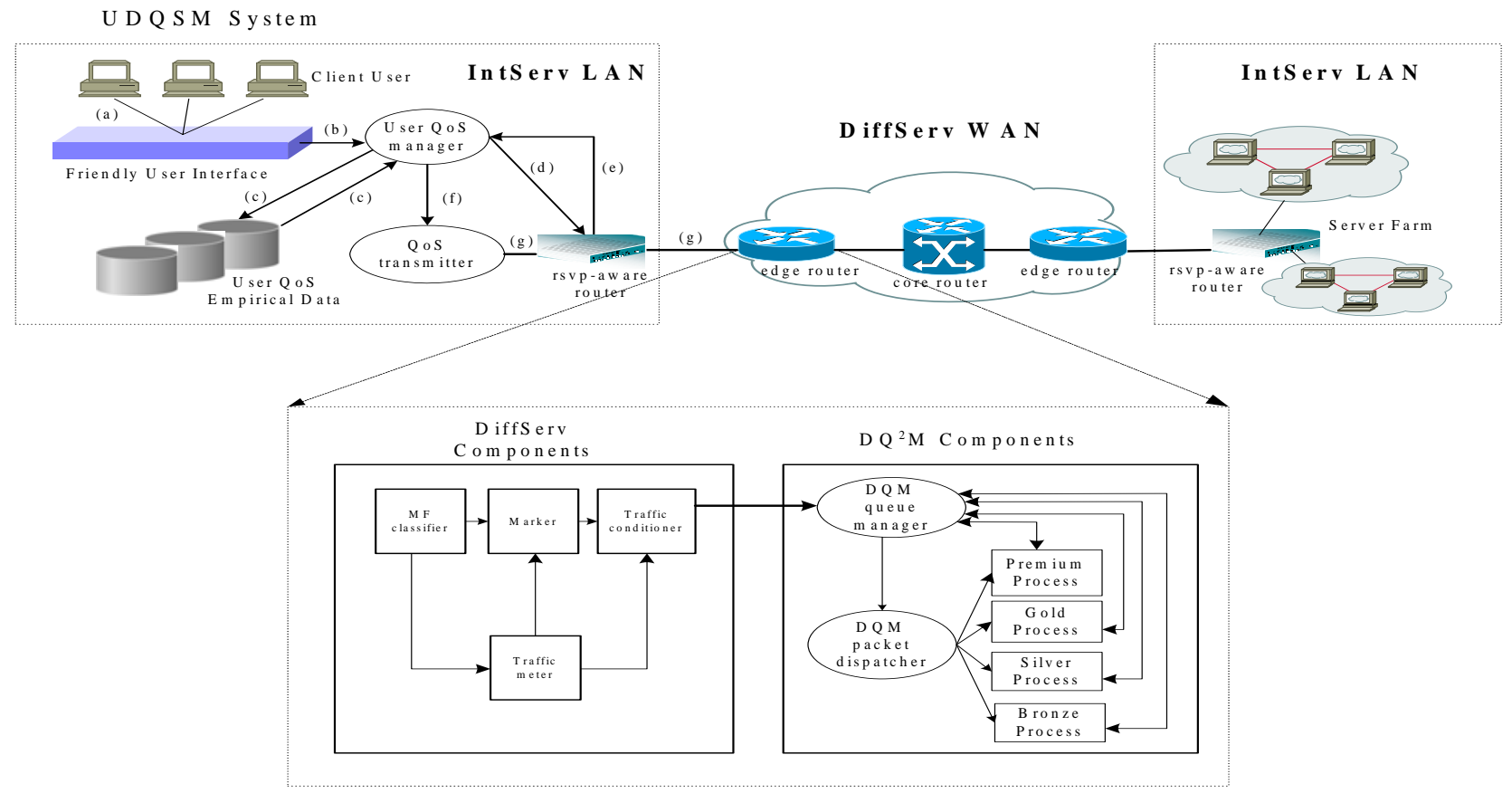

Fig. 2 Dynamic user-oriented QoS framework architecture and system components.

(1) Friendly User Interface. It provides a user-oriented QoS specification tool, which has three QoS specification modes for different QoS specification behavior mentioned in Section 2. For user-oriented dynamic QoS specification, the tool also provides two quality buttons for users to increase and decrease the presentation quality dynamically.

(2) User QoS Manager. It achieves the dynamic QoS mapping between the application and network layer. The user QoS manager derives an adequate QoS contract for the network layer from the user's abstraction QoS requirements and from the user QoS empirical database. The user QoS empirical database is divided into four service regions, which are the premium, gold, silver, and bronze services. The user QoS manager determines the adequate QoS values by matching the user QoS requirement with the empirical data in the database. The derived adequate QoS contract is sent to the RSVP-aware router to obtain the admitted QoS contract after the admission control.

(3) QoS Transmitter. It is responsible for transmitting the media packets according to the admitted QoS contract.

(4) Empirical Database. It stores user empirical data derived from historical sessions and is represented as
Experienced_Value and Admitted_Value. The representation format is $\left\{\left(B_{e}, D_{e}, J_{e}\right),\left(B_{a}, D_{a}, J_{a}\right)\right\}$. The former item is Experienced_Value composed of three metrics (bandwidth, delay, jitter), which are measured and stored at the client. It represents the practical QoS traffic experienced by the user. The latter item is Admitted_Value, which represents the admitted resources in the RSVP router. Depending on the modes of the user interface, there are different ways to store the empirical data. As depicting in Figure 3, all of the empirical data is divided into four regions corresponding to the four kinds of service types in the Easy mode. For the Qualitative mode, the four regions of Figure 3 are divided into three intervals corresponding to the qualitative items in each QoS parameters. There is no classification of the empirical data in the quantitative mode. Table 3 and Table 4 depict the interval value in the Easy mode regions and the interval in the Qualitative mode regions, respectively. 


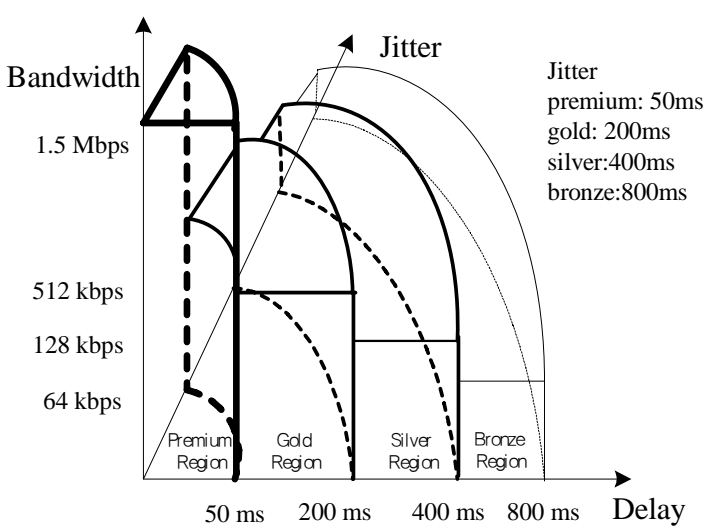

Fig. 3 Regions in empirical database.

Table 3: The interval value in the Easy mode regions

\begin{tabular}{|c|c|c|c|}
\hline Service Type & Bandwidth & Delay & Jitter \\
\hline Premium & (1.5M, 512K) & (0ms, 50ms) & (0ms, 50ms) \\
\hline Gold & $(128 \mathrm{~K}, 512 \mathrm{~K})$ & (50ms, 200ms) & (50ms, 200ms) \\
\hline Silver & $(64 \mathrm{~K}, 128 \mathrm{~K})$ & (200ms, 400ms) & $(200 \mathrm{~ms}, 400 \mathrm{~ms})$ \\
\hline Bronze & $(4 \mathrm{~K}, 64 \mathrm{~K})$ & (400ms, $800 \mathrm{~ms})$ & $(400 \mathrm{~ms}, 800 \mathrm{~ms})$ \\
\hline
\end{tabular}

Table 4: The interval value in the Easy mode regions

\begin{tabular}{|c|c|c|c|c|}
\hline \multicolumn{2}{|c|}{$\begin{array}{l}\text { Parameter } \\
\text { Service Type }\end{array}$} & Bandwidth & Delay & Jitter \\
\hline \multirow[t]{3}{*}{$\mathrm{P}$} & High & (1.5M, 1171k) & (0ms, 16ms) & (0ms, 16ms) \\
\hline & Medium & $(1171 \mathrm{~K}, 842 \mathrm{~K})$ & (16ms, 33ms) & (16ms, 33ms) \\
\hline & Low & $(841 \mathrm{~K}, 512 \mathrm{~K})$ & (33ms, 50ms) & (33ms, 50ms) \\
\hline \multirow[t]{3}{*}{ G } & High & $(384 \mathrm{~K}, 512 \mathrm{~K})$, & (50ms, 100ms) & (50ms, 100ms) \\
\hline & Medium & (256K, 384K) & (100ms, 150ms) & $(100 \mathrm{~ms}, 150 \mathrm{~ms})$ \\
\hline & Low & (256K, 128K) & $(150 \mathrm{~ms}, 200 \mathrm{~ms})$ & $(150 \mathrm{~ms}, 200 \mathrm{~ms})$ \\
\hline \multirow[t]{3}{*}{$\mathrm{S}$} & High & $(128 \mathrm{~K}, 107 \mathrm{~K})$ & (200ms, 266ms) & (200ms, 266ms) \\
\hline & Medium & $(107 \mathrm{~K}, 85 \mathrm{~K})$ & (266ms, 333ms) & (266ms, 333ms) \\
\hline & Low & $(85 \mathrm{~K}, 64 \mathrm{~K})$ & (333ms, 400ms) & (333ms, 400ms) \\
\hline \multirow[t]{3}{*}{ B } & High & $(64 \mathrm{~K}, 44 \mathrm{~K})$ & (400ms, 533ms) & (400ms, 533ms) \\
\hline & Medium & $(44 \mathrm{~K}, 24 \mathrm{~K})$ & (533ms, 667ms) & (533ms, 667ms) \\
\hline & Low & $(24 \mathrm{~K}, 4 \mathrm{~K})$ & $(667 \mathrm{~ms}, 800 \mathrm{~ms})$ & (667ms, 800ms) \\
\hline
\end{tabular}

As depicting in Figure2, the operation scenario of the User-oriented Dynamic QoS Specification and Mapping (UDQSM) system is described as follows.

(A) A user specifies the required QoS parameters via the QoS specification tool.

(B) The user QoS manager obtains a pre-adequate QoS contract from the QoS specification tool.

(C) The manager determines the adequate QoS contract by matching the user QoS requirement with the empirical data in the database.
(D) The derived adequate QoS values are sent to the RSVP router for the admission control of resource reservation.

(E) The RSVP router responses the admitted QoS values after the admission control to the user QoS manager.

(F) The manger sends the admitted QoS values to the QoS transmitter.

(G) The transmitter and the RSVP router transmit the media packets according the admitted QoS contract.

During the media transmission, a user can dynamically push the quality upgrade/downgrade button to find a satisfactory presentation quality. Whenever the user makes a new QoS request at runtime, the manager renegotiates with the RSVP-aware router to determine the new adequate and admitted QoS values. However, we refer $[3,11]$ to the mapping issues between the DiffServ and IntServ that is out of the scopes.

Figure 4 depicts the detailed procedures to determine the most adequate QoS values. Firstly, the User QoS Manager matches the request value to the empirical data. There are three policies to determine the request value.

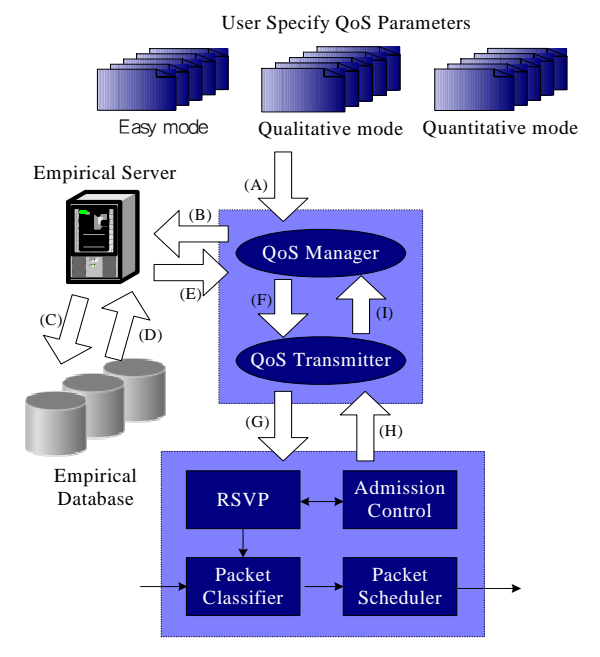

Fig. 4 The dynamic QoS specification based on UDQF.

(1) Maximum Policy. Find the most frequent admitted value in the empirical data as the request value.

(2) Aggressive Policy. Based on the four divided regions in the empirical database, the request value is the highest value of the region. For example, if the user selects the premium service, the request value is (1.5Mbps, 0ms, 10ms). 


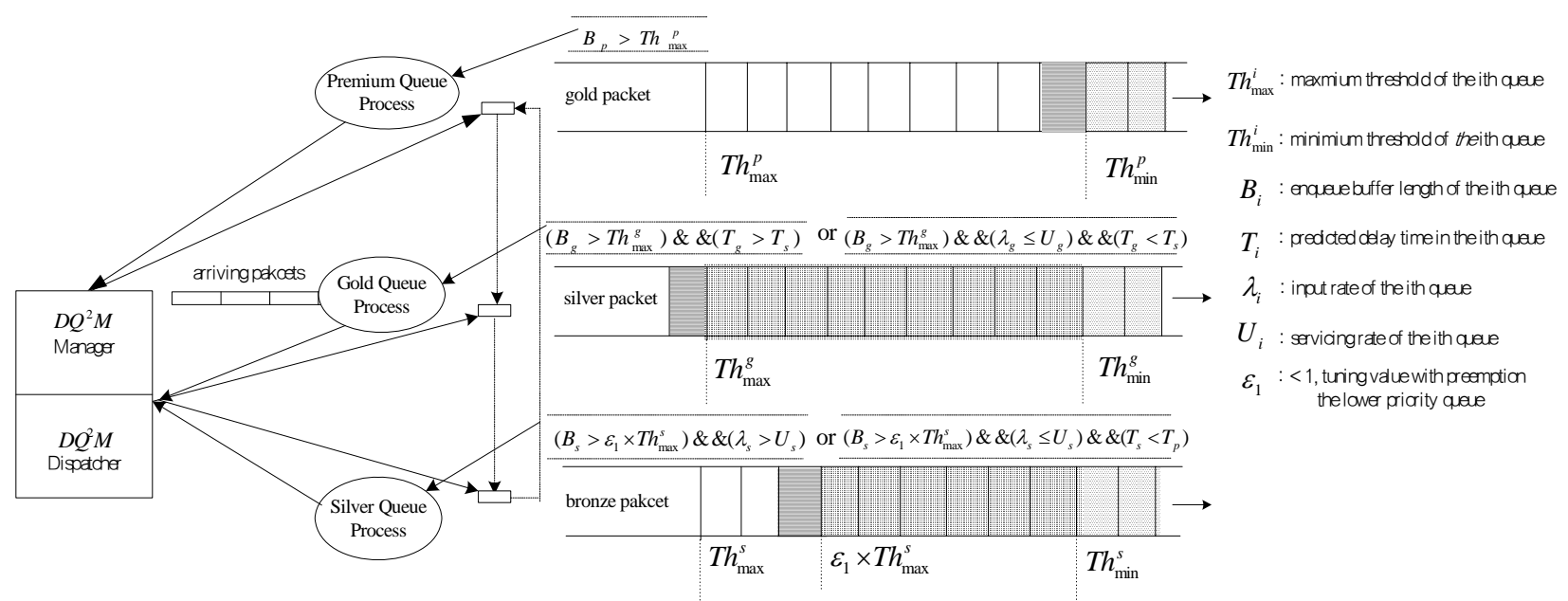

Fig. 5 The system architecture of $\mathrm{DQ}^{2} \mathrm{M}$ for the gold service.

(3) Conservative Policy. Contrast to the aggressive policy, the conservative policy employs the lowest value of the region. For example, if the user selects the premium service, the request value is $(512 \mathrm{Kbps}, 50 \mathrm{~ms}$, $50 \mathrm{~ms}$ ). When the request value is obtained, Empirical Server finds the best matched experienced value, which is derived from Equation (1). The admitted value of this matched experienced value is then referred to the adequate value for the resource reservation signaling value.

$$
\begin{aligned}
& \underset{1<i \leq n_{j}}{\operatorname{Min}\left\{Q_{i}\right\}} \\
& Q_{i}=\sqrt{\left(B_{r}-B_{i}\right)^{2}+\left(D_{r}-D_{i}\right)^{2}+\left(L_{r}-L_{i}\right)^{2}}
\end{aligned}
$$

$Q_{i}$ : The QoS distance between the request value and experienced value of the $i_{\text {th }}$ empirical datum.

$B_{i}, D_{i}, L_{i}$ : Bandwidth, delay, and jitter of the experienced value of the $i_{\text {th }}$ empirical data.

$B_{r}, D_{r}, L_{r}$ : Bandwidth, delay, and jitter of the request value.

$n_{j}$ : The number of the empirical data in the $j_{\text {th }}$ region.

There are some problems occurred due to the matched empirical value. In some cases, an over-offered empirical value may be produced due to a long-term burst, which induces a terrible experienced value. Under this situation, the adequate value for the admission control of UDQF will exceed user's expected quality too much. It induces that the inefficient utilization of the router resources and the rejection probability of the admission control increases. The Equation (1) is modified not only to determine an empirical value but also retrieves any compatible value given from the corresponding $Q_{i}$ under a heuristic value. The most reasonable admitted value in the compatible data is the adequate value for signaling.

\section{Dynamic QoS Queue Mapping Algorithm}

The objective of Dynamic QoS Queue Mapping (DQ²M) algorithm is to adapt the traffic patterns of each priorities queue to avoid heavy traffic of one queue while others incur starvation of packets. As depicting in Figure 5, $\mathrm{DQ}^{2} \mathrm{M}$ mechanism is composed of the $\mathrm{DQ}^{2} \mathrm{M}$ Manager, the $\mathrm{DQ}^{2} \mathrm{M}$ Dispatcher, and the Queue Process for each service queue.

The $\mathrm{DQ}^{2} \mathrm{M}$ is based on the RED mechanism and definitely co-operates with WFQ. The $\mathrm{DQ}^{2} \mathrm{M}$ policy applies the thresholds of RED mechanism in each queue as the indicator of traffic condition. When the average length of a queue is lower than the minimum threshold, this queue is in starvation state. When the average length of a queue exceeds the maximum threshold, the queue is in congestion state. The $\mathrm{DQ}^{2} \mathrm{M}$ Manager determines whether to re-dispatch the arriving packets to other queues or not based on the status of thresholds in each queue. The $\mathrm{DQ}^{2} \mathrm{M}$ Dispatcher dispatches the arriving packets to a queue according to the decision of the $\mathrm{DQ}^{2} \mathrm{M}$ Manager. 
The detailed $\mathrm{DQ}^{2} \mathrm{M}$ steps for the arriving packet, which belongs to the gold service queue, are described in Figure 5 , described as follows.

(1) When the gold_queue_process detects a congestion state by the threshold_indication, the arriving packet is held and the gold_queue_process notifies the $\mathrm{DQ}^{2} \mathrm{M}$ Manager.

(2) The $\mathrm{DQ}^{2} \mathrm{M}$ Manager determines the gold packet to preempt the resources of the silver queue.

(3) The $D^{2} M$ Manager notifies the silver_queue_process to determine the threshold status the silver queue.

(4) If the silver queue isn't in congestion state, this packet is marked as silver service DSCP value and sent to the silver queue by the $\mathrm{DQ}^{2} \mathrm{M}$ Dispatcher.

(5) If the silver queue does not have enough resources to serve this packet and there is no lower priority queue to be preempted, the $\mathrm{DQ}^{2} \mathrm{M}$ Manager notifies the premium_queue_process to determine the threshold status of the premium queue.

(6) When the average length of the premium queue is lower than the minimum threshold, this packet is dispatched to the premium queue.

(7) If the condition above doesn't exist, the original gold packet is dispatched to the gold queue again.

In Figure 5, Equation (2) determines the value of delay time prediction. The value of is used to adapt the threshold of the lower priority queue. When the high priority packets are re-dispatched to low priority queue, the maximum threshold of low priority queue should be decreased to avoid the high priority packet incur too much delay.

$$
T_{i}=\frac{Q_{i}(K)}{U_{i}(K)-\lambda_{i}(K)}
$$

\section{Conclusions}

With the dynamic essence of the network traffic and user ambiguous QoS requirements, a well-designed multimedia QoS system should adapt the request resource reservation according to the user QoS requirements and the network resource status. In this paper, by integrating IntServ (LAN) and DiffServ (WAN) network environment, we proposed the Dynamic User-Oriented QoS Framework (UDQF) to achieve dynamic QoS specification and mapping according to the user perceivable satisfaction. UDQF provides the User-oriented Dynamic QoS Specification and Mapping (UDQSM) system to adaptively achieve QoS specification and mapping at the application layer. A user-friendly interface for QoS parameter authoring is designed in UDQSM system. In the DiffServ of DUQF, we proposed the Dynamic QoS Queue Mapping ( $\left.\mathrm{DQ}^{2} \mathrm{M}\right)$ algorithm to avoid the inefficient resource utilization originating from the DiffServ that a popular service queue suffers congestion and the other queues are in the starvation state. The $\mathrm{DQ}^{2} \mathrm{M}$ policy can enhance the utilization of the overall network resources and decrease the packets lost ratio.

\section{Acknowledgments}

The authors would like to thank the National Science Council of Republic of China, Taiwan, for financially supporting this research under Contract No. NSC 942218-E-020-001.

\section{References}

[1] C., Aurrecoechea, A.T., Campbell and L., Hauw, "A survey of QoS architectures”, Multimedia Systems, pp.138-151, 2000.

[2] A., Bouch, M.A., Sasse and H., DeMeer, "Of Packets and People: A User-centered Approach to Quality of Service”, IEEE IWQOS, pp.189 -197, 2003.

[3] T., Chahed, G., Hebuterne and C., Fayet, "On Mapping of Loss and Delay Between Intserv and Diffserv", IEEE ECUMN 2000, pp. 235-249, 2000.

[4] F., Cheong and R., Lai, "QoS specification and mapping for distributed multimedia systems: A survey of issues", The Journal of Systems and Software, vol. 45, pp.127-139, 2000.

[5] C. Dovrolis and P., Ramanathan, "A Case for Relative Differentiated Services and the Proportional Differentiation Model”, IEEE Network, vol.13, pp.26-34, 1999.

[6] G., Ghinea, J.P., Thomas and R.S., Fish, "Quality of Perception to Quality of Service Mapping Using a Dynamically Reconfigurable Communication System", Global Telecommunications Conference, pp.2061-2065, 2004.

[7] C.C. Li, S.L. Tsao, M.C. Chen, Y.L. Sun and Y.M. Huang, "Proportional Delay Differentiation Service Based on Weighted Fair Queuing”, Proceedings of IEEE Ninth International Conference on Computer Communications and Networks, pp.418-423, 2002.

[8] C., Metz, "RSVP: General-Purpose Signaling for IP”, IEEE Internet Computing vol.3 Iss.3, pp.95-99, 1999.

[9] L.A. Dasilva, "QoS Mapping along the Protocol Stack: Discussion and Preliminary Results", IEEE International Conference on Communications vol.2, pp.713 -717, 2003.

[10] K., Nahrstedt, Xu, Dongyan, D., Wichadakul and Li, Baochun, "QoS-Aware Middleware for Ubiquitous and 
Heterogeneous Environments”, IEEE Communications Magazine, vol.39, Issue: 11, pp. 140 -148, 2001.

[11] H.C., Su and R.H., Hwang, "Multicast Provision in a Differentiated Services Network", Proceedings.of International Conference on Information Networking, pp.189 -196, 2005.

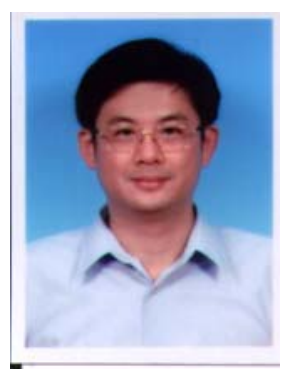

Hsu-Yang Kung received his BS degree from Tatung University, MS degree from National Tsing-Hwa University, PhD degree from National Cheng-Kung University, Taiwan, all in computer science and information engineering. He is currently an associate professor at the Department of Management Information Systems, National Pingtung University of Science and Technology, Taiwan. His research interests include distributed multimedia systems, wireless and mobile communications, and the embedded multimedia applications.

Fu-Wen Kuo received the Master degree form National Pingtung University of Science and Technology majoring in Management Information Systems in Taiwan. His research interests include embedded communication software and distributed multimedia systems. 